\title{
Assessment of Common Turbulence Models under Conditions of Temporal Acceleration in a Pipe
}

\author{
A. Khaleghi ${ }^{1}$, M. Pasandideh-Fard ${ }^{1}$, M. Malek-Jafarian ${ }^{2}$ and Y.M. Chung ${ }^{3}$ \\ ${ }^{1}$ Department of Mechanical Engineering, Ferdowsi University, Mashhad 91775-1111, Iran \\ ${ }^{2}$ Department of Mechanical Engineering, Birjand University, Birjand, 97175-376, Iran \\ ${ }^{3}$ School of Engineering \& Centre for Scientific Computing, University of Warwick, Coventry CV4 7AL, UK
}

Email: khaleghi79@yahoo.com

(Received January 23, 2009; accepted May 20, 2009)

\begin{abstract}
In this paper, transient flow in a pipe at Reynolds numbers (based on bulk velocity and diameter) ranged from 7000 to 45200 is numerically simulated using four common turbulence models. The models considered are the BaldwinLomax algebraic model, the $\kappa-\varepsilon$ model with wall correction of Lam and Bremhorst, the $\kappa-\omega$ model and the $\kappa-\varepsilon-v^{2}$ model of Durbin. The results of these models are compared with those of the recent experiments reported in the literature. The predicted velocity and delay period using the models compared well with measured values for short and long ramp-up flow excursions. The delay period of the calculated turbulence kinetic energy close to the pipe centerline is around $4 \mathrm{sec}$ which agrees with the experiments. The $\kappa-\varepsilon-v^{2}$ model was found to provide the best results compared to the measured data in the region away from the wall. At the end of the excursion near the wall, however, the results of this model differs from those of the experiments.
\end{abstract}

Keywords: Turbulence model, Pipe flow, Unsteady, DNS, URANS.

\section{NOMENCLATURE}

$\begin{array}{ll}C_{\mu} & \text { Constant of } \kappa-\varepsilon \text { model } \\ D & \text { pipe Diameter } \\ F_{\text {kleb }} & \text { Klebanoff factor in B-L turbulence model } \\ F_{\max } & \text { Max. wake function in turbulence model } \\ F_{\text {wake }} & \text { wake function in B-L turbulence model } \\ l_{\text {mix }} & \text { Mixing length scale in turbulence model } \\ P & \text { pressure } \\ r & \text { radius of the pipe } \\ R e & \text { Reynolds Number(based on bulk velocity ) } \\ R e_{0} & \text { initial Reynolds Number } \\ R e_{1} & \text { final Reynolds Number } \\ R e_{\tau} & \text { Re Number(based on friction velocity) } \\ t & \text { time } \\ T I & \text { turbulence intensity } \\ u_{\tau} & \text { friction velocity } \\ U_{c} & \text { centerline pipe velocity }\end{array}$

\section{INTRODUCTION}

The study of unsteady turbulent pipe flow is of value in providing information which can lead to an improved understanding of the turbulence phenomenon. In such flows certain fundamental aspects of turbulent flow are exposed, which although present in steady turbulent flows, are not apparent under such conditions. In

$\begin{array}{ll}U_{b} & \text { bulk velocity } \\ U_{b 0} & \text { initial bulk velocity } \\ U_{b 1} & \text { final bulk velocity } \\ u & \text { velocity of fluid } \\ x & \text { axial direction } \\ y_{w} & \text { distance from the wall } \\ \varepsilon & \text { dissipation rate } \\ \gamma & \text { Dimensionless ramp rate parameter } \\ \kappa & \text { Turbulence kinetic energy } \\ \rho & \text { Density } \\ \tau & \text { Shear stress } \\ v & \text { Kinematic viscosity } \\ v_{t} & \text { Eddy viscosity } \\ \omega & \text { Vorticity } \\ + & \text { Superscript for dimensionless parameters }\end{array}$

addition, due to the effect of inertia, some additional features of turbulence specific to transient flows can be present. Due to the technical difficulties involved, detailed measurements of turbulence in transient flow were not possible until quite recently. As a result of the availability of modern instrumentation and powerful computers, transient turbulent flow can now be readily investigated. Therefore, these flows are getting more 
attention for research studies (He and Jackson, 2000). Unsteady turbulent pipe flows can be conveniently classified into two groups, namely periodic pulsating flows and non-periodic transient flows. Here we will describe the latter category briefly which in this article we will examine it.

\subsection{Non-Periodic Turbulent Flows}

In contrast to pulsating pipe flow, non-periodic transient pipe flow has received relatively little attention. The few studies of this kind undertaken so far have involved a variety of types of imposed excursions of flow rate. Kataoka et al. (1975) studied the start-up response to a step input of flow rate in a pipe. The study of Maruyama et al. (1976) was concerned with transient turbulent pipe flow following a stepwise increase of flow rate from an initial steady turbulent flow condition. Delays were observed in the response of turbulence, which were found to be greater for the centre of the pipe than the region close to the wall. Kurokawa and Morikawa (1986) studied flow transients with gradually increasing and decreasing flow rate in a pipe. Their study showed that the transition Reynolds number increased with ramp up rate and that, even for a very small imposed acceleration, transition from laminar to turbulent flow was significantly postponed. The experimental study of Lefebvre (1987) was concerned with accelerating flow in a pipe. Discussion was mainly concentrated on transition from laminar to turbulent flow based on results from single excursions. The mean velocity profiles and turbulent intensity profiles both generally exhibited a quasi-steady variation, although a reduction in turbulence intensity was clearly evident at the beginning of some of the transients. In the He and Jackson (2000) investigation, unsteady flow development was studied, following a 'stepwise' increase in flow rate. Turbulence was generated initially in the near-wall region and subsequently propagated towards the centre of the pipe. More detailed investigation, involving ramp-up type increases in velocity, identified delays associated with turbulence production, energy redistribution and radial propagation of turbulence were reported. Finally, Greenblatt and Moss (2004) measured fully developed turbulent pipe flows subjected to temporal pressure gradients larger than those considered previously. Velocity profiles were initially characterized by significant reduction of their wake component. The final phase of the acceleration was characterized by reconstitution of the wake, producing a velocity profile inflection and the generation of turbulence in that vicinity.

The fact that turbulence is out of equilibrium and that the relaminarization and retransition can take paths dependent on the frequency constitutes a severe challenge for conventional Unsteady Reynolds Averaged Navier-Stokes (URANS) models. However, most of the models used in the literature are extensions of steady eddy-viscosity closures with different recipes to compute the eddy viscosity (Gundogdu and Carpinlioglu, 1999a, 1999b). Thus, a better understanding of the capabilities and limitations of URANS models is required. Direct, Large-eddy and experimental simulation data can be very useful in helping to achieve this goal.

Hsu et al. (2000) used Large Eddy Simulation (LES) to validate a RANS model in the purely oscillating case. Scotti and Piomelli (2002) compared the performance of four well-established turbulence models for the unsteady Reynolds-averaged Navier-Stokes equations to the flow in a channel driven by a pressure gradient oscillating around a nonzero mean. The results were compared with those of experiments, DNS and LES. The $\kappa-\varepsilon-v 2$ model was found to be generally superior to the other models considered. Chung and Malek-Jafarian (2005) performed direct numerical simulation for a turbulent flow subjected to a sudden change in pressure gradient. Four turbulence models were tested in their study to compare the DNS data and URANS models. They found that Baldwin-Lomax model is not suitable for unsteady flow calculations. On the other hand one and two equation models gave reasonable results in the near wall region. Recently, Yorke and Coleman (2004) used the results of direct numerical simulation for a $\mathrm{Re}_{\tau}=390$ channel flow subjected to the strain and deceleration typical of Adverse Pressure Gradients (APGs), to the point of skin-friction reversal, as a reference for comparing four simple turbulence models. They found that the model accuracy varied significantly.

The purpose of this study is to compare the predictions of four well-known turbulence models applied to the flow in a pipe with results from a recent experimental work of He and Jackson (2000). The statistics satisfy a one-dimensional unsteady problem and contain many of the complications associated with transient pipe flow, thus allowing a straight-forward but nontrivial assessment of the models for pipe flow, with defined boundary and initial conditions and an acceptable range of Reynolds number.

\section{TurbulenCE MOdels}

Because of the simple geometry, the URANS equations for the horizontal pipe in non-dimensional for simply become as:

$$
\begin{aligned}
& u^{+}=\frac{u}{u_{\tau}}, x^{+}=\frac{u_{\tau}}{v} x, y^{+}=\frac{u_{\tau}}{v} y \\
& p^{+}=\frac{p}{\rho u_{\tau}^{2}}, t^{+}=\frac{u_{\tau}^{2}}{v} t, \quad v^{+}=\frac{v_{t}}{v} \\
& \operatorname{Re}_{\tau}=\frac{u_{\tau}}{v} R \\
& \frac{\partial u^{+}}{\partial t^{+}}=-\frac{d p^{+}}{d x^{+}}+\frac{\partial}{\partial y^{+}}\left(\left(1+v_{t}^{+}\right) \frac{\partial u^{+}}{\partial y^{+}}\right) \\
& (j+1) \frac{\tau}{R}=-\frac{d p}{d x} j=1 \text { for cylinderical Sys. } \\
& -\frac{d p^{+}}{d x^{+}}=\frac{v}{R u_{\tau}}=\frac{1}{\operatorname{Re}_{\tau}}
\end{aligned}
$$


In comparison with other works (Scotti and Piomelli, 2002; Yorke and Coleman, 2004) where at most four different models for turbulence were considered, in this study, we have examined almost four commonly relative URANS models, too. In order of increasing computational complexity they are the Baldwin-Lomax (BL) algebraic model (Baldwin and Lomax, 1987) the standard $\kappa-\varepsilon$ model with the wall correction of Lam and Bremhorst (1981), the $\kappa-\omega$ model of Wilcox (1988) and the $\kappa-\varepsilon-v^{2}$ model of Durbin (1995). All models assume the existence of the eddy viscosity which is used to express the Reynolds stress as:

$$
-\overline{u^{\prime} v^{\prime}}=v_{t} \frac{\partial u}{\partial r}
$$

where $v_{t}$ denotes the eddy viscosity. Next, we briefly review each model.

\subsection{Baldwin-Lomax (BL)}

The Baldwin-Lomax model (Baldwin and Lomax, 1987) is a two-layer algebraic zero-equation model which gives the eddy viscosity, $\mu_{t}$, as a function of the local boundary layer velocity profile. The model is suitable for high-speed flows with thin attached boundary-layers, typically present in aerospace and turbomachinery applications. This model is commonly used in quick design iterations where robustness is more important than capturing all details of the flow physics. The Baldwin-Lomax model is not suitable for cases with large separated regions and significant curvature/rotation effects. Non-dimensional form of Baldwin-Lomax turbulence model is:

$$
\begin{aligned}
& v_{t i}=l_{\text {mix }}^{2}|\omega| \\
& l_{\text {mix }}=k y^{+}\left[1-e^{-y^{+} / A_{0}^{+}}\right] \\
& \omega^{+}=\frac{\partial u^{+}}{\partial y^{+}} \\
& v_{\text {to }}=\alpha C_{c p} F_{\text {wake }} F_{k l e b} \\
& F_{\text {wake }}=\min \left[y_{\max } F_{\max }, \frac{C_{w k} y_{\max }^{+} u_{\text {dif }}^{+2}}{F_{\max }}\right] \\
& F_{\max }=\frac{1}{k}\left[\max \left(l_{\max }|\omega|\right)\right] \\
& \left.F_{k l e b}=\left[1+5.5\left(\frac{y^{+}}{y_{\max }^{+}} C_{k l e b}\right)^{6}\right]^{-1}\right]^{-1} \\
& k=0.41, \alpha=0.0168, A_{0}^{+}=26 \\
& C_{c p}=1.6, C_{k l e b}=0.3, C_{w k}=1.0
\end{aligned}
$$

$y_{\max }$ is the value of $y$ at which $\boldsymbol{l}_{\max } .|\omega|$ achieves its maximum value. $u_{d i f}$ is the maximum value of $u$ for boundary layers. For free shear layers, $u_{d i f}$ is the difference between the maximum velocity in the layer and the value of $\mathrm{u}$ at $\mathrm{y}=\mathrm{y}_{\max }$.

\section{$2.2 \kappa-\varepsilon$ Two-Equation model}

The $\kappa-\varepsilon$ model has become one of the most popular turbulence models used in simulations for many practical applications. The model determines the turbulence kinetic energy $\kappa$ and its dissipation rate $\varepsilon$ by the transport relations. Non-dimensional form of $\kappa-\varepsilon$ turbulence model is:

$$
\begin{aligned}
& k^{+}= \frac{k}{u_{\tau}^{2}}, \varepsilon^{+}=\frac{v}{u_{\tau}^{4}} \varepsilon \\
& v_{t}^{+}=C_{\mu} f_{\mu} k^{+2} / \varepsilon^{+} \\
& R= k^{+2} / \varepsilon^{+} \\
& f_{1}= 1.45, f_{2}=1-0.3 e^{-R^{2}}, f_{\mu}=e^{\frac{-2.5}{1+R / 50}} \\
& \frac{\partial k^{+}}{\partial t^{+}}=v_{t}^{+}\left(\frac{\partial u^{+}}{\partial y^{+}}\right)^{2}-\varepsilon^{+}-2\left(\frac{\partial \sqrt{k^{+}}}{\partial y^{+}}\right)^{2} \\
& \quad+\frac{\partial}{\partial y^{+}}\left[\left(1+\frac{v_{t}^{+}}{\sigma_{k}}\right) \frac{\partial k^{+}}{\partial y^{+}}\right] \\
& \frac{\partial \varepsilon^{+}}{\partial t^{+}}=C_{\varepsilon 1} f_{1} v_{t}^{+} \frac{\varepsilon^{+}}{k^{+}}\left(\frac{\partial u^{+}}{\partial y^{+}}\right)^{2}-C_{\varepsilon 2} f_{2} \frac{\varepsilon^{+2}}{k^{+}} 2 v_{t}^{+}\left(\frac{\partial^{2} u^{+}}{\partial y^{+2}}\right)^{2} \\
& \quad+\frac{\partial}{\partial y^{+}}\left[\left(v+\frac{v_{t}^{+}}{\sigma_{\varepsilon}}\right) \frac{\partial \varepsilon^{+}}{\partial y^{+}}\right] \\
& c_{\varepsilon_{1}}= 1.45, c_{\varepsilon_{2}}=0.18, c_{\mu}=0.09, \sigma_{k}=1, \\
& \sigma_{\varepsilon}= 1
\end{aligned}
$$

Many formulations of this model exist such as LaunderSharma, Chien, Lam-Bremhorst, Shih-Mansour and Nagano-Tagawa models. Our choice is the model proposed by Lam-Bremhorst (1981), because in this model it is not required to find the instantaneous $u_{\tau}$.

\section{$2.3 \kappa-\omega$ Two-Equation model}

This model proposed by Wilcox (1988), does not use wall damping functions. Non-dimensional form of $\kappa-\omega$ turbulence model is:

$$
\begin{aligned}
& k^{+}=\frac{k}{u_{\tau}^{2}}, \omega^{+}=\frac{v}{u_{\tau}^{2}} \omega \\
& v_{t}^{+}=\frac{k^{+}}{\omega^{+}} \\
& \frac{\partial k^{+}}{\partial t^{+}}=v_{t}^{+}\left(\frac{\partial u^{+}}{\partial y^{+}}\right)-\beta^{*} k^{+} \omega^{+}+\frac{\partial}{\partial y^{+}}\left[\left(1+\sigma^{?} v_{t}^{+}\right) \frac{\partial k^{+}}{\partial y^{+}}\right] \\
& \frac{\partial \omega^{+}}{\partial t^{+}}=\alpha v_{t}^{+} \frac{\omega^{+}}{k^{+}}\left(\frac{\partial u^{+}}{\partial y^{+}}\right)^{2}-\beta \omega^{+2}+\frac{\partial}{\partial y^{+}}\left[\left(1+\sigma v_{t}^{+}\right) \frac{\partial \omega^{+}}{\partial y^{+}}\right] \\
& \alpha=\frac{5}{9}, \beta=\frac{3}{40}, \beta^{*}=0.09 \\
& \sigma=0.5, \sigma^{*}=0.5
\end{aligned}
$$

Where $\omega$ is the vorticity, for which a transport equation (written for $\omega$ ) is given, including the effects of production, diffusion and dissipation. Unlike any other two-equation models, the $\kappa-\omega$ model does not involve damping functions and allows simple Dirichlet boundary conditions to be specified. Because of its simplicity, the $\kappa-\omega$ model is superior to other models, especially with regards to numerical stability. However, the $\kappa-\omega$ model (like many other models) does not correctly predict the asymptotic behavior of the turbulence for the region close to the wall. In addition, the $\kappa-\omega$ model does not accurately represent $\kappa$ and $\varepsilon$ distribution in agreement with DNS data (Moser, 1999). 


\section{4. $\kappa-\varepsilon-v^{2}$ model}

The $\kappa-\varepsilon-v^{2}$ model proposed by Durbin (1995) represents an extension of the standard $\kappa-\varepsilon$ model. This model enables the correct kinematic boundary condition to be imposed on the normal component of turbulent intensity. Hence, wall blocking is represented. The virtue of this model is that arbitrary "damping functions" are not required. The eddy viscosity is taken to be:

$v_{t}^{+}=C_{\mu} v^{+2} T$

The Non-dimensional form of $\kappa-\varepsilon-v 2$ turbulence model proposed by Durbin is as:

$$
\begin{aligned}
& \frac{\partial k^{+}}{\partial t^{+}}=P^{+}-\varepsilon^{+}+\frac{\partial}{\partial y^{+}}\left[\left(1+\frac{v_{t}^{+}}{\sigma_{k}}\right) \frac{\partial k^{+}}{\partial y^{+}}\right] \\
& \frac{\partial \varepsilon^{+}}{\partial t^{+}}=C_{\varepsilon 1}^{*+} \frac{P^{+}}{T^{+}}-C_{\varepsilon 2} \frac{\varepsilon^{+}}{T^{+}}+\frac{\partial}{\partial y^{+}}\left[\left(1+\frac{v_{t}^{+}}{\sigma_{\varepsilon}}\right) \frac{\partial \varepsilon^{+}}{\partial y^{+}}\right] \\
& f_{22}^{+}=\frac{v}{u_{\tau}^{2}} f_{22} \quad, \quad v^{+2}=\frac{v^{2}}{u_{\tau}^{2}} \\
& T^{+}=\max \left[\frac{k^{+}}{\varepsilon^{+}}, 6\left(\frac{1}{\varepsilon^{+}}\right)^{\frac{1}{2}}\right] \\
& \frac{\partial v^{+2}}{\partial t^{+}}=k^{+}+f_{22}^{+}-v^{+2} \frac{\varepsilon^{+}}{k^{+}}+\frac{\partial}{\partial y^{+}}\left[\left(1+\frac{v_{t}^{+}}{\sigma_{k}}\right) \frac{\partial v^{+2}}{\partial y^{+}}\right] \\
& L^{+2} \frac{\partial^{2} f_{22}^{+}}{\partial y^{+2}}-f_{22}^{+}=\left(1-C_{1}\right) \frac{\left(\frac{2}{3}-\frac{v^{+2}}{k^{+}}\right)}{T^{+}}-C_{2} \frac{P^{+}}{k^{+}} \\
& L^{+}=C_{L} \max \left[\frac{k^{+\frac{3}{2}}}{\varepsilon^{+}}, C_{\eta}\left(\frac{1}{\varepsilon^{+}}\right)^{\frac{1}{4}}\right] \\
& P^{+}=v_{t}^{+}\left(\frac{\partial u^{+}}{\partial y^{+}}\right)^{2}, C_{\varepsilon 1}^{*_{+}}=C_{\varepsilon 1}\left(1+a_{1} \frac{P^{+}}{\varepsilon^{+}}\right) \\
& c_{\varepsilon_{1}}=1.44, c_{\varepsilon_{2}}=1.9, c_{\mu}=0.23, \sigma_{k}=0.9 \text {, } \\
& \sigma_{\varepsilon}=1.3, a_{1}=0.1, c_{1}=1.3, c_{2}=0.3, c_{L}=0.2, c_{\eta}=90
\end{aligned}
$$

Where $v$ is a velocity scale (in pipe geometry $v^{2}$ represents the intensity of radial fluctuations).

\section{NUMERICAL ASPECTS}

The URANS equation (2) and the relevant model equations are solved using a finite-difference timemarching code. The code employs the second-order Crank-Nicolson discretization on a non-uniform grid (stretched in the $r$ direction) including the range of turbulence models, mentioned above. Because we invoke symmetry, therefore we solve the momentum and model equations over the half-channel or pipe domain and impose zero-slope boundary conditions at the centerline on $\mathrm{u}$ and the model transport variables. We utilize 400 grid points between the wall and the channel or pipe centerline for each model. A geometric grid stretching monotonically clusters the points near the wall, with the first grid point 0.1 initial wall units above the wall for all four models. The time step of
$5 * 10^{-2}$ initial wall units was chosen sufficiently small for all models. Therefore the results are grid independent with these values of grid and time step.

\section{Results}

\subsection{Evaluation with DNS data}

Evaluation of the models accuracy can be performed a priori by computing the velocity profile, Reynolds stresses and the other properties using the 'true' velocity field represented here by the DNS data of Moser et al. at $\operatorname{Re}_{\tau}=590$ (Moser, 1999).

Figures 1 and 2 show the corresponding modeled mean velocity profiles for different models and compared to the DNS channel flow data (the solid curve).

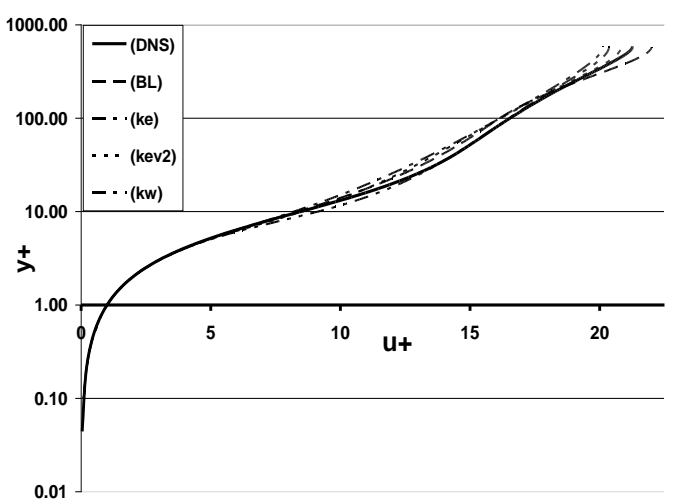

Fig. 1. DNS and model predictions of $u^{+}$

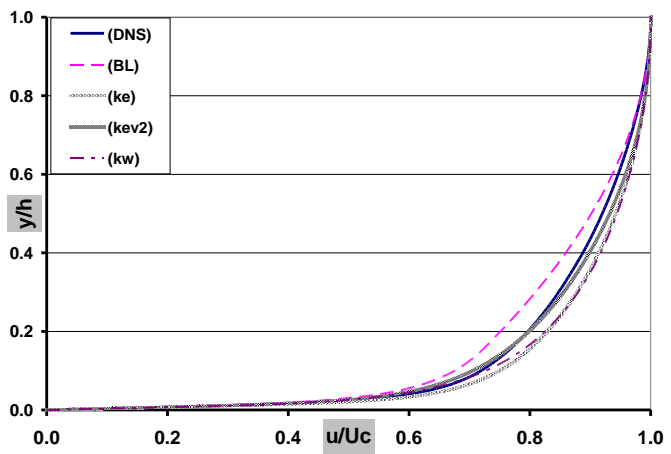

Fig. 2. DNS and model predictions of $\bar{u} / U_{c}$

Figure 1 is a log-linear plot to show the wall region in more detail and is in wall units. On the other hand, Fig. 2 is a linear plot. All the models agree well with each others in the region close to the wall. Beyond $\mathrm{y}^{+}=10$ all profiles do not agree with each others. Predictions of the $\kappa-\varepsilon-v^{2}$ model give the best overall agreement for $\mathrm{u}^{+}$and $\bar{u} / U_{c}$ compared with the DNS, when $u_{\tau}$ and $U_{c}$ normalizations are considered, respectively. The BL model overestimates the velocity profile (Fig. 2).

Away from the wall, the exaggerated wake assumed by the BL closure is responsible for its consistent underprediction of the (smaller-wake) channel flow profile, which is emphasized by the $U_{c}$ normalization used in 
Fig. 2 (Yorke and Coleman, 2004). The other model predictions like $\kappa-\varepsilon$ and $\kappa-\omega$ are very close to each others.

Profile of Reynolds shear stresses are displayed in Fig. 3. Agreement of all model predictions with the DNS data seems to be almost perfect, except BL and $\kappa$ $\omega$ models. The BL results are under-estimated and the $\kappa-\omega$ results are over-estimated. This comparison allows us to evaluate the validity of the modeling analysis in itself, removing possible errors that can be caused by the modeling of the terms in the equations.

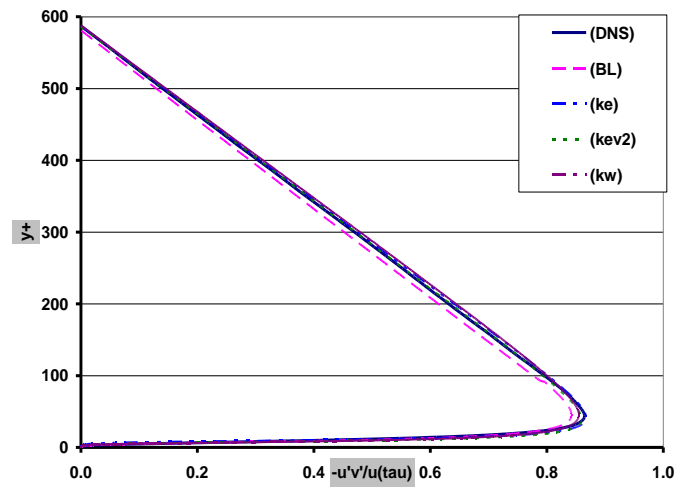

Fig. 3. DNS and model predictions of $-u^{\prime} v^{\prime} / u_{\tau}^{2}$

The distribution of eddy viscosity of each model is shown in Fig. 4. They agree closely with each other and DNS data near the wall, especially for $\mathrm{y}_{\mathrm{w}} / \mathrm{h}<0.1$. Large discrepancies occur in the outer layer. The BL model is under predicted but the one- and two-equation $v_{t}$ predictions are over predicted in the outer-layer. All of them differ from the DNS data by as much as $93 \%$ (for $\kappa-\omega$ model) or higher in particular at the centerline. The BL $v_{t}$ is about $20 \%$ smaller than the DNS data in the outer layer. The maximum discrepancy is for $\kappa-\omega$, and the other eddy viscosities fall roughly half-way between the $\kappa-\omega$ and DNS profiles.

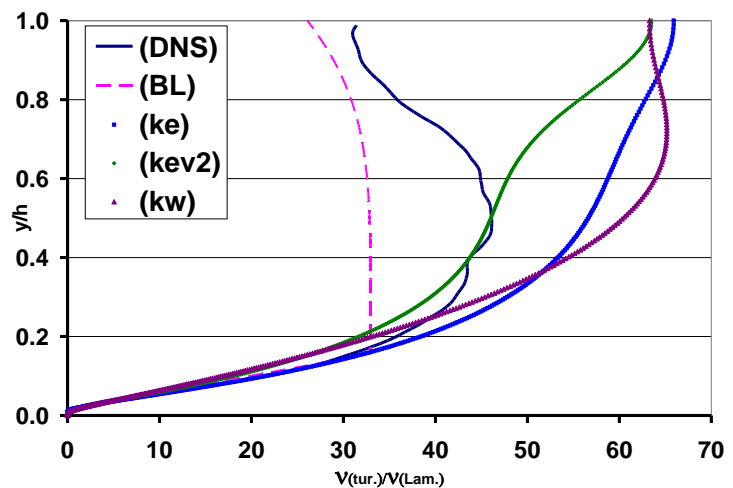

Fig. 4. DNS and model predictions of $v_{\mathrm{T}} / v$

Figures 5-7 show the budgets computed from the DNS data and $\kappa-\varepsilon$ model for the turbulent kinetic energy in local wall coordinates. The k-budget is largely dominated by dissipation and diffusion near the wall. While, away from the wall the production and dissipation terms are dominant. Turbulence diffusion term changes sign at $\mathrm{y}^{+}=7$.
At the wall the dissipation rate balances the diffusion rate. There is a good agreement between the production term in all model equations and k-budget from the DNS (Figs. 5-7). But the large discrepancies of the $\kappa-\varepsilon$ and $\kappa$ $\omega$ models compared to DNS for both the dissipation and diffusion terms are taken into account (Figs.5 and 7).

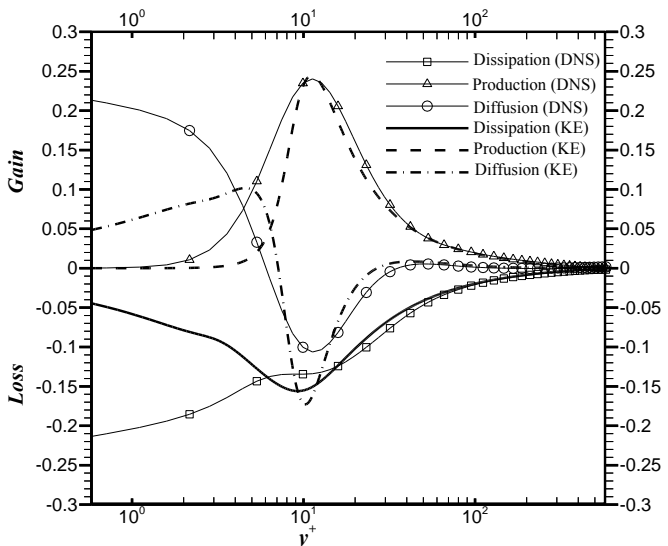

Fig. 5. DNS and $\boldsymbol{k}-\boldsymbol{\varepsilon}$ model predictions of turbulence kinetic energy budget

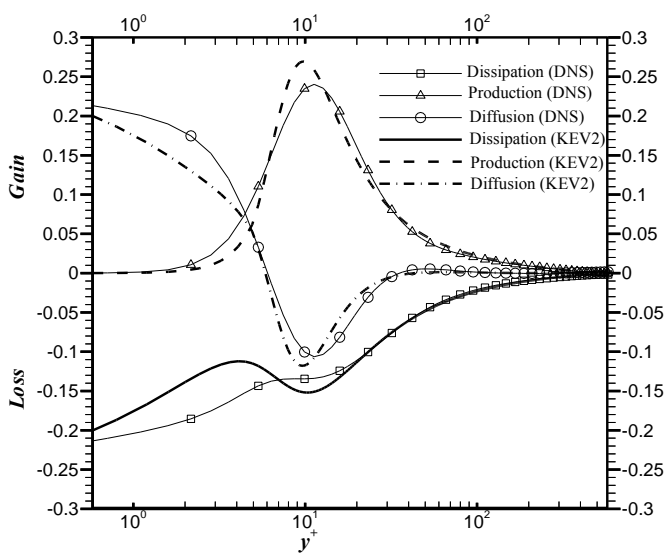

Fig. 6. DNS and $\kappa-\varepsilon-v^{2}$ model predictions of turbulence kinetic energy budget

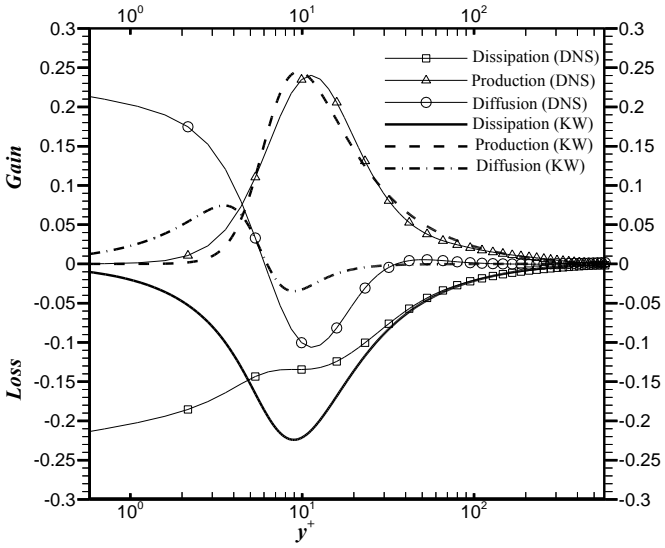

Fig. 7. DNS and $\kappa-\omega$ model predictions of turbulence kinetic energy budget

$\kappa-\varepsilon-v^{2}$ model shows the best prediction for dissipation, production and diffusion terms in comparison with DNS data (Fig. 6). In the next section we will 
demonstrate the performance of these models for unsteady turbulent flow in a pipe.

\section{COMPARING WITH EXPERIMENTS}

$\mathrm{He}$ and Jackson studied the accelerating and decelerating ramp-type turbulent flows in a pipe $(\mathrm{He}$ and Jackson, 2000). The ramp-up experiments were performed in which the ramp rate $\mathrm{dU}_{\mathrm{b}} / \mathrm{dt}$ was varied by imposing excursions of flow rate during which the bulk velocity increased linearly with time from an initial value $\mathrm{U}_{\mathrm{b} 0}=0.138 \mathrm{~m} / \mathrm{s}$ to a final value $\mathrm{U}_{\mathrm{b} 1}=0.891 \mathrm{~m} / \mathrm{s}$ in periods of time which ranged from $2 \mathrm{sec}$ to $90 \mathrm{sec}$. The corresponding initial and final Reynolds numbers $\left(\mathrm{Re}_{0}\right.$ and $\mathrm{Re}_{1}$ ) were constant at the values 7000 and 45200 . Therefore the Reynolds numbers based on wall units and $\mathrm{u}_{\tau}\left(\operatorname{Re}_{\tau}\right)$ are equal to 240.5 and 1243. But the dimensionless ramp rate parameter $\gamma=D / U_{b o} \cdot\left(1 / U_{\tau 0} \cdot d U_{b} / d t\right)$ increased systematically from 0.34 (pseudo-steady flow) to 15.3 (unsteady turbulent flow). Their experimental results not only showed how mean flow and turbulence respond to imposed transients but also provided new insight into turbulence dynamics. We now compare the predictions of the URANS models with the experimental data of $\mathrm{He}$ and Jackson mentioned above for 45 and 5 seconds ramp-up flow excursions.
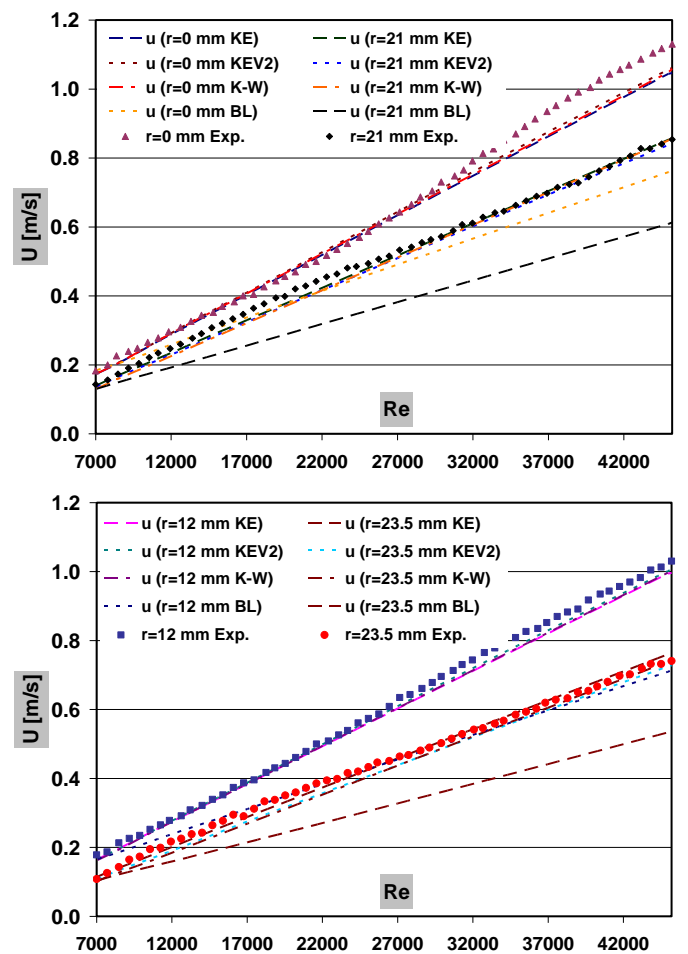

Fig. 8. Experiment and turbulence models predictions of local mean velocity in a $45 \mathrm{sec}$ time period ramp-up flow excursion

Figure 8 shows the development of local mean velocity for $45 \mathrm{sec}$ ramp-up flow excursion $(\gamma=0.68)$. The results are plotted as a function of Reynolds number for a number of radial positions. The response of the local velocity in core and wall regions is different. The performance of $\mathrm{BL}$ model is poor and very under- estimated for prediction of the turbulence transient flow at these conditions. The agreement between the other models and experiments are excellent for all radial positions, except in the core. All the models tend to under-estimate the velocity during the acceleration at centerline.

The results for a ramp-up excursion of flow rate with a time period of $5 \sec (\gamma=6.1)$ is shown in Fig. 9. The BL model significantly under-predicts the velocity. The results from all the other models are very close to each other and essentially match the experiments, except in the core region $(\operatorname{Re}>32000)$ at the end of the acceleration stage where the results differ from those of the experiments. But the $\kappa-\varepsilon-\nu^{2}$ predictions are very close to experiments at these conditions $(\mathrm{r}=0$ and $\mathrm{Re}>32000)$.
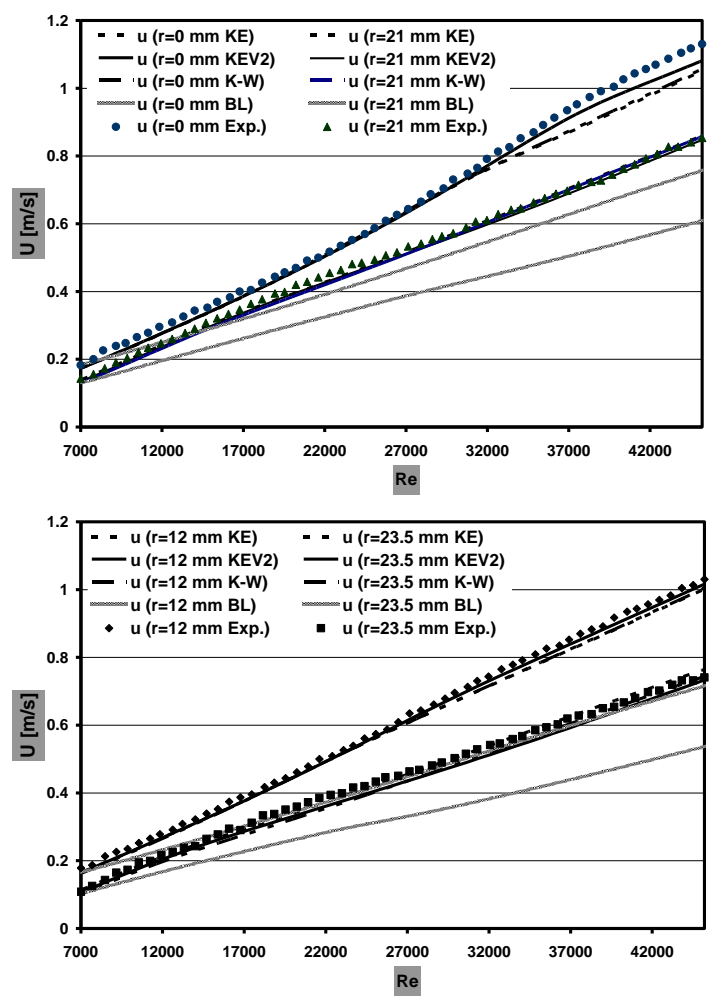

Fig. 9. Experiment and turbulence models predictions of local mean velocity in a $5 \mathrm{sec}$ time period ramp-up flow excursion

The development of the turbulence kinetic energy (k) during a 5 sec ramp-up flow excursion is shown in Fig. 10 for several radial positions. As can be seen, there is a delay effect to the imposed flow transient in the response of velocity fluctuations. All of the models predict the delay effect as well as experiment. The response for the excursion with a marked change is very slow in the core region; however the response becomes faster near the wall $(\mathrm{r}=23.5 \mathrm{~mm})$. He and Jackson (2000) defined the delay period $(\tau)$ as "the period of time from the start of an excursion to the point at which the faster response starts". As can be seen, this parameter is clearly a function of radial position. It is less than $1 \mathrm{sec}$ near the wall. At the centre it approaches $4 \mathrm{sec}$. The response of turbulence kinetic energy to the imposed excursion starts in the wall region and is 
transmitted towards the centre of the pipe, at a certain speed (He and Jackson, 2000). At all positions except near the wall (at Re>25000), $\kappa-\varepsilon-v^{2}$ significantly agrees with experiments, for the entire ramp-up excursion. Whereas $\kappa-\omega$ agree only at high Reynolds number with experiment near the wall $(\mathrm{r}=21,23.5 \mathrm{~mm})$. But they underestimate the turbulent kinetic energy at low Reynolds number $(\operatorname{Re}<25000) . \kappa-\varepsilon$ model predictions are poor at the end of flow excursion in the core $(\mathrm{Re}>32000, \mathrm{r}=0 \mathrm{~mm})$.
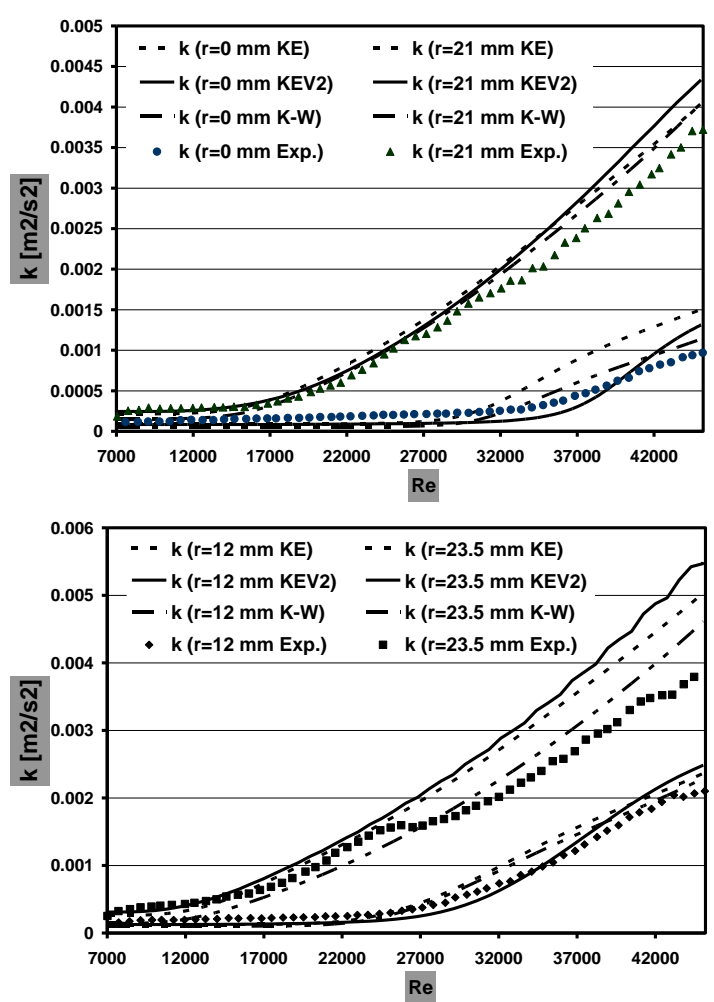

Fig. 10. Experiment and turbulence models predictions of kinetic energy in a $5 \mathrm{sec}$ time period ramp-up flow excursion

The development of the turbulence intensity $T I=\sqrt{2 k / 3} / U_{b}$ in $5 \mathrm{sec}$ time period ramp-up excursion is plotted in Fig. 11. The turbulence intensity is attenuated in accelerating transient flow. The $\mathrm{k}$ distribution (Fig. 11) remains at or near to their initial values at the beginning of excursion (delay period). On the other hand, the bulk velocity increases at this time. So the reduction in turbulence intensity is a consequence of the delayed response of the turbulence quantities. Near the wall $(\mathrm{r}=21,23.5 \mathrm{~mm}) \kappa-\varepsilon-\nu^{2}$ and $\kappa-$ $\varepsilon$ predictions are poor when Reynolds number increases, whereas $\kappa-\omega$ model agree better than $\kappa-\varepsilon-v^{2}$ and $\kappa-\varepsilon$. But the $\kappa-\varepsilon-\nu^{2}$ and $\kappa-\varepsilon$ predictions are good at the beginning of excursion and vice-versa for $\kappa-\omega$. Away from the wall $(\mathrm{r}=0,12 \mathrm{~mm}), \kappa-\varepsilon-v^{2}$ has a good agreement with experiments. The other models are more under-predicting or over-predicting. The reason that $\kappa-\varepsilon-v^{2}$ could not predict the turbulent intensity accurately near the wall is related to the prediction of $\mathrm{k}$ (turbulence kinetic energy). It derives from the above definition $T I=\sqrt{2 k / 3} / U_{b}$. However, $\kappa-\varepsilon-v^{2}$ predicts well the turbulent kinetic energy (Fig. 10), near the wall at the region of delay time $(\operatorname{Re}<10000)$. After that the discrepancies appear. Therefore, these discrepancies develop to the turbulent intensities (See the TI definition).
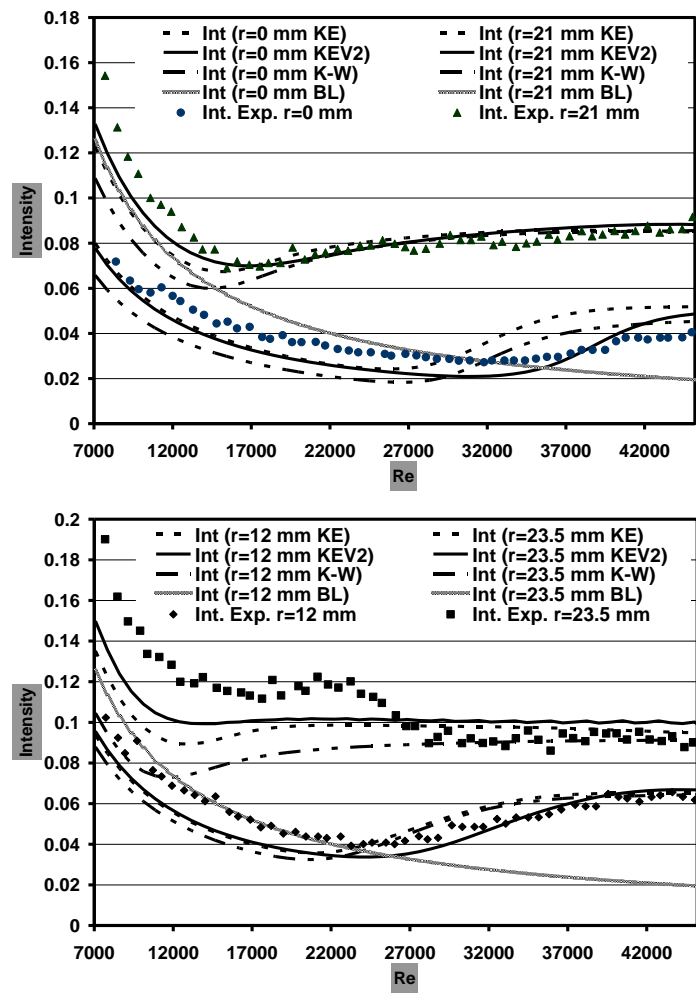

Fig. 11. Experiment and turbulence models predictions of turbulence intensity in a $5 \mathrm{sec}$ time period ramp-up flow excursion

The development of turbulent shear stress in the $5 \mathrm{sec}$ ramp-up flow excursion is shown in Fig. 12 for various radial positions. This Figure exhibits the features of delay and propagation. The development of the turbulent shear stress is similar to that of turbulent kinetic energy (Fig. 10). The delay is dependent on the distance from the wall at positions in the core region. But in the wall region turbulent shear stress exhibits a distinct delay for a period which is independent of the location, and then it builds up. BL predictions are poor, but the other models predict the correct behavior during the acceleration, especially away from the wall $(r=0,12$, $21 \mathrm{~mm})$. Near the wall and at the beginning of acceleration, all models predict the experiment data as well. But the models predictions overestimate the shear stress after the acceleration continue. Of the four models, $\kappa-\varepsilon-v^{2}$ appear to be the most accurate.

If we pay more attention to the way of calculation $v_{t}$ in $\kappa-\varepsilon-v^{2}$ model, it can be inferred that it is inherently different with $\kappa-\varepsilon, \kappa-\omega$ and $\mathrm{BL}$ models for the region close to the wall. This is because of the effect of $y$ component of velocity employed in this region. Furthermore, there is a term including $\partial \sqrt{k} / \partial y$ with minus sign in the k-equation of $\kappa-\varepsilon$ model, which does not exist in $\kappa-\varepsilon-v^{2}$ model. Therefore, in the $\kappa-\varepsilon$ model, the amount of $\mathrm{k}$ will be much less than that of the $\kappa-\varepsilon$ $v^{2}$ model for the points adjacent to the wall, where $\mathrm{y}$ gradients of the properties are very high. However, for 
the region close to the pipe centre where y gradients of all properties diminish, the two models approach each other. Furthermore, the $\varepsilon$ equation of the two $\kappa-\varepsilon$ and $\kappa-$ $\varepsilon-v^{2}$ models are only different in the term including $\partial u^{2} / \partial y^{2}$ added in the $\kappa-\varepsilon-v^{2}$ model. It is clear that this term will be disappeared after a distance from the wall, and the two models approach each other.

He and Jackson (2000) found that the delays are more or less the same for all cases at a particular position despite the imposed acceleration being very different. Their calculation showed that the responses at the centre of the pipe deviate slightly after a mean value of about $4 \mathrm{sec}$. A comparison of the responses of turbulent kinetic energy at the centre of the pipe for various ramp-up flow excursions is shown in Fig. 13.
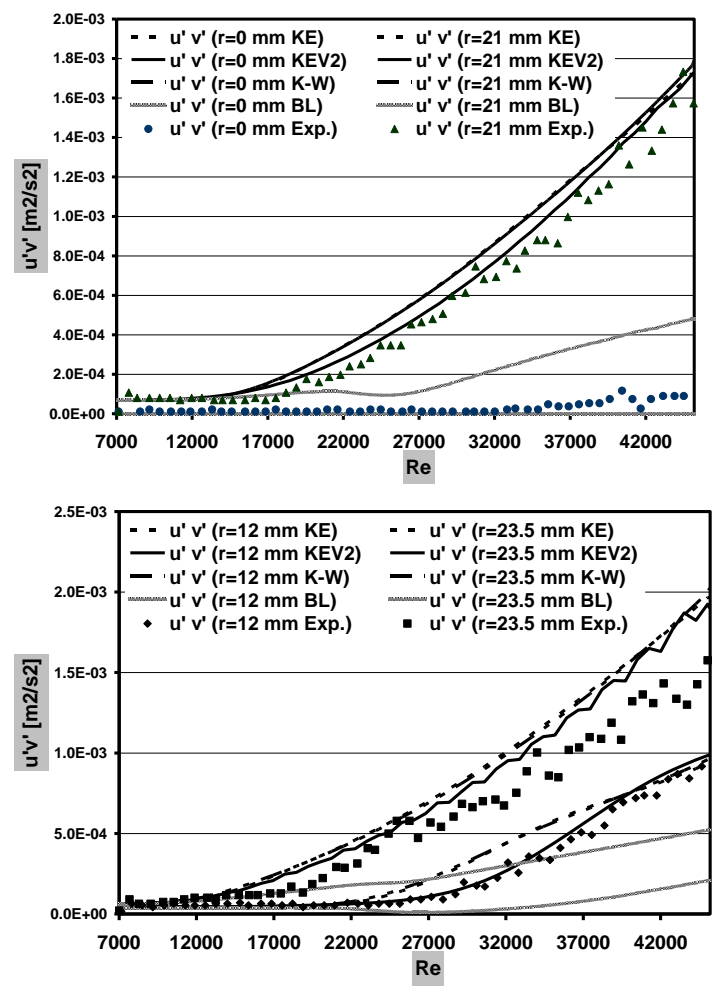

Fig. 12. Experiment and turbulence models predictions of turbulent shear stress in a $5 \mathrm{sec}$ time period ramp-up flow excursion

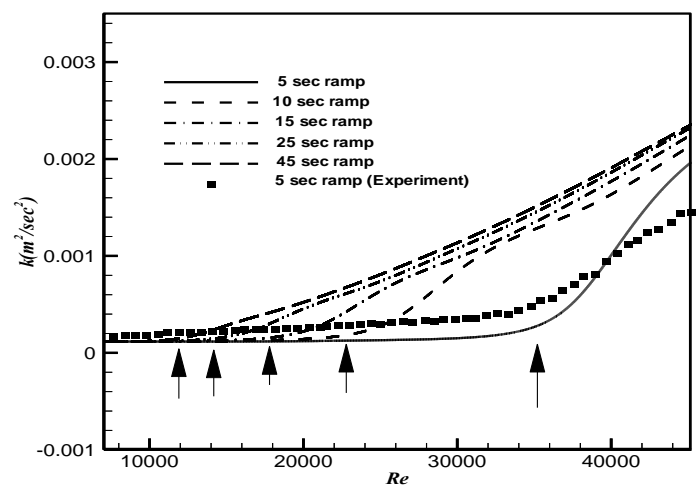

Fig. 13. Comparison of the responses of turbulent kinetic energy calculated with $\kappa-\varepsilon-v^{2}$ model for various ramp rates at pipe centre
This figure presents accurate calculations of $\kappa-\varepsilon-v^{2}$ model. The change-over points of the response curves are indicated with arrows. The absolute delays for numerical and experimental data of $\mathrm{He}$ and Jackson are given in Table 1. As observed, the $\kappa-\varepsilon-\nu^{2}$ predictions and experiments deviate after a mean value of about 4 sec. This deviation is little for experiments and is a little bit more for numerical predictions at some ramp time periods, like 5, 25 and $45 \mathrm{sec}$.

Table 1 Absolute delay at the centre of the pipe

\begin{tabular}{|l|c|c|c|c|c|}
\hline Ramp time period $(\mathrm{sec})$ & 5 & 10 & 15 & 25 & 45 \\
\hline Dimensionless ramp rate $(\gamma)$ & 6.1 & 3.1 & 2 & 1.5 & 0.68 \\
\hline Delay $\tau(\mathrm{sec})-$ experiments & 3.9 & 4.1 & 4.0 & 3.9 & 4.0 \\
\hline $\begin{array}{l}\text { Delay } \tau(\mathrm{sec})-\kappa-\varepsilon-\nu^{2} \\
\text { predictions }\end{array}$ & 3.78 & 3.91 & 4.11 & 3.58 & 4.7 \\
\hline
\end{tabular}

\section{CONCLUSIONS}

Temporal accelerating transient flow in a pipe was simulated using four different RANS turbulence models. Simulation results were compared with those of DNS data (Moser, 1999) and the experiments performed by $\mathrm{He}$ and Jackson (2000). The models considered are the Baldwin-Lomax algebraic model, the $\kappa-\varepsilon$ model with wall correction of Lam and Bremhorst, the $\kappa-\omega$ model of Wilcox, and the $\kappa-\varepsilon-v^{2}$ model of Durbin.

To evaluate the accuracy of these models, the velocity profile, Reynolds stresses and the other properties at $\operatorname{Re}_{\tau}=590$ were calculated using the 'true' velocity field represented by the DNS data (Moser, 1999). It was shown that the $\kappa-\varepsilon-v^{2}$ model has the potential for giving a fairly accurate estimate of the k-budget, velocity profile and Reynolds stress.

The results of BL model did not agree well with experimental results. All of the other models predicted the velocity and the delay period close to those of the experiment for short and long ramp-up flow excursions. The delay period of the calculated turbulence kinetic energy in the core region was around $4 \mathrm{sec}$ which agreed with the experiments. The models accuracy varied widely. The $\kappa-\varepsilon-v^{2}$ scheme gave the best overall agreement with the measured data of kinetic energy and turbulence shear stress, in the region away from the wall $(\mathrm{r}=0,12,21 \mathrm{~mm})$. The $\kappa-\varepsilon-v^{2}$ results deviated from experiment at the end of excursion near the wall $(\mathrm{r}=23.5$ $\mathrm{mm})$.

\section{REFERENCES}

Baldwin, B.S. and H. Lomax (1978). Thin-layer approximation and algebraic model for separated turbulent flows. AIAA Paper, 78-257.

Chung, Y.M. and M. Malek-Jafarian (2005). Direct Numerical Simulation of Unsteady Decelerating Flows. Fourth International Symposium on Turbulence and Shear Flow Phenomena (TSFP-4), Williamsburg, USA.

Durbin, P.A. (1995). Separated flow computations with the $\kappa-\varepsilon-v^{2}$ model. AIAA J. 33, 659-664. 
Greenblatt, D. and E. Moss (2004). Rapid temporal acceleration of a turbulent pipe flow. J. Fluid Mech. 514, 65-75.

Gundogdu, M.Y. and M.O. Carpinlioglu (1999a). Present state of art on pulsatile flow theory (part 1: laminar and transitional flow regimes). Japanese Society of Mechanical Engineering International Journal Series B(42), 384-397.

Gundogdu, M.Y. and M.O. Carpinlioglu (1999b). Present state of art on pulsatile flow theory (part 2: laminar and transitional flow regimes). Japanese Society of Mechanical Engineering International Journal Series B(42), 398-410.

He, S. and J.D. Jackson (2000). A study of turbulence under conditions of transient flow in a pipe. $J$. Fluid Mech. 408, 1-38.

Hsu, C.T., X. Lu and M.K. Kwan (2000). LES and RANS studies of oscillating flows over flat plate. American Society of Chemical Engineering Journal of Engineering Mechanics 126, 186-193.

Kataoka, K., T. Kawabata and K. Miki (1975). The start-up response of pipe flow to a step change in flow rate. J. Chem. Engng. Japan 8, 266-271.

Kurokawa, J. and M. Morikawa (1986). Accelerated and decelerated flows in a circular pipe ( $1^{\text {st }}$ report, velocity profiles and friction coefficient). Bull. JSME 29, 758-765

Lam, C.K.G. and K.A. Bremhorst (1981). Modified form of the $\kappa-\varepsilon$ model for predicting wall turbulence. Journal of Fluids Engineering 103, 456-460.

Lefebvre, P.J. (1987). Characterization of Accelerating Pipe Flow. PhD thesis, University of Rhode Island.

Maruyama, T., T. Kuribayashi and T. Mizushina (1976). The structure of the turbulence in transient pipe flows. J. Chem. Engng. Japan 9, 431-439.

Moser, R.D., J. Kim and N.M. Mansour (1999). Direct numerical simulation of turbulent channel flows up to $\mathrm{Re}_{\tau}=590$. Physics of Fluids 11, 943-945.

Scotti, A. and U. Piomelli (2002). Turbulence models in pulsating flows. AIAA J. 40, 537-540.

Wilcox, D.C. (1988). Reassessment of the scaledetermining equation for advanced turbulence models. AIAA Journal 26, 1299-1310.

Yorke, C.P. and G.N. Coleman (2004). Assessment of common turbulence models for an idealized adverse pressure gradient flow. European Journal of Fluid Mechanics B/Fluids 23, 319-337. 